\title{
Hernia paracecal, reto diagnóstico y quirúrgico: reporte de caso y revisión de la literatura
}

\author{
Paracecal hernia, diagnostic and surgical challenge: \\ case report and literature review
}

\author{
Carolina María Rodríguez ${ }^{1} \mathbb{D}$, Eduardo Bayter $^{2} \mathbb{D}$, Rogers Leonardo Baquero ${ }^{3} \mathbb{D}$
}

Médica, especialista en Cirugía General; instructora asociada, Fundación Universitaria Ciencias de la Salud, Hospital de San José,
Bogotá, D.C., Colombia
2 Médico, especialista en Cirugía General, adscrito, Hospital de San José, Bogotá, D.C., Colombia
3 Médico, residente de Cirugía General, Fundación Universitaria Ciencias de la Salud, Hospital de San José, Bogotá, D.C., Colombia

\section{Resumen}

Introducción. La hernia paracecal es una entidad rara que corresponde al $13 \%$ de todas las hernias internas. Aunque algunas pueden ser adquiridas, pero la mayoría son congénitas y se originan en los recesos creados durante la adherencia y fusión de los repliegues peritoneales alrededor de la región ileocecal.

Caso clínico. Se trata de un hombre de 42 años de edad a quien se le diagnosticó una apendicitis aguda. La intervención quirúrgica se inició con una incisión para apendicectomía localizada; no obstante, por los hallazgos intraoperatorios, se decidió la conversión a laparotomía y se produjo el hallazgo incidental de una hernia paracecal.

Resultados. El paciente fue hospitalizado y, en el quinto día postoperatorio, presentó obstrucción intestinal por lo cual fue intervenido nuevamente; se encontró escaso líquido de reacción peritoneal y adherencias. Se le dio el egreso hospitalario cuatro días después de su segundo procedimiento quirúrgico y continuaba asintomático hasta su último control.

Discusión. Las hernias paracecales son hernias internas que se describen como la protrusión de una víscera hueca por una apertura mesentérica o peritoneal. Sus manifestaciones clínicas son variadas y se puede presentar sin síntomas, con dolor abdominal localizado o hasta con obstrucción intestinal. El tratamiento quirúrgico se basa en liberar y examinar el segmento intestinal herniado y, de requerirse, proceder a la resección con anastomosis; se debe resecar el saco herniario cuando sea evidente, valorar los vasos mesentéricos y cerrar el defecto herniario. Se trata de una enfermedad poco común y es importante conocer su manejo con la finalidad de evitar complicaciones, ya que continúa siendo un reto diagnóstico para el cirujano.

Palabras clave: hernia abdominal; embriología; peritoneo; tracto gastrointestinal; intestino delgado; cirugía.

Fecha de recibido: 26/03/2019 - Fecha de aceptación: 26/05/2020

Correspondencia: Carolina María Rodríguez, Calle 10 N 18-75, Hospital de San José, segundo piso, oficina de Cirugía General, Bogotá, D.C., Colombia. Teléfono: (321) 280-6339

Correo electrónico: cmrodriguez2@fucsalud.edu.co

Citar como: Rodríguez CM, Bayter E, Baquero RL. Hernia paracecal, reto diagnóstico y quirúrgico, reporte de caso y revisión de la literatura. Rev Colomb Cir. 2020;35:500-6. https://doi.org/10.30944/20117582.777

Este es un artículo de acceso abierto bajo una Licencia Creative Commons - BY-NC-ND https://creativecommons.org/licenses/by-ncnd/4.0/deed.es 


\begin{abstract}
Introduction: Paracecal hernia is a rare entity that represents $13 \%$ of all internal hernias. Although some may be acquired, most are congenital and originate in the recesses created during the adhesion and fusion of peritoneal folds around the ileocecal region.

Clinical case: This is a 42-year-old man who was diagnosed with acute appendicitis. The surgical intervention began with an incision for localized appendectomy; however, due to intraoperative findings, conversion to laparotomy was decided and the incidental finding of a paracecal hernia occurred. The patient was hospitalized and, on fifth postoperative day presented intestinal obstruction, for which he was reoperated; little peritoneal reaction fluid and adhesions were found. He was discharged four days after his last surgical procedure and remained asymptomatic until his last control.

Discussion: Paracecal hernias are internal hernias that are described as the protrusion of a hollow viscera through a mesenteric or peritoneal opening. Its clinical manifestation are varied and can present without symptoms, with localized abdominal pain or even intestinal obstruction. Surgical management is based on releasing and examining the herniated intestinal segment and, if required, proceed with resection with anastomosis. The hernia sac should be resected when evident, assess the mesenteric vessels and close the hernia defect. It is a rare pathology and it is important to know its management in order to avoid complications, as it continues to be a diagnostic challenge for the surgeon.
\end{abstract}

Key words: hernia, abdominal; embryology; peritoneum; gastrointestinal tract; intestine, small; surgery.

\section{Introducción}

Las hernias internas son relativamente raras; entre ellas, la hernia paracecal tiene una incidencia particularmente baja ${ }^{\mathrm{I}}$, reportada hasta en el $13 \%^{2,3}$. Las hernias internas pueden originarse en la formación de aperturas peritoneales a partir de traumas o de procedimientos quirúrgicos previos ${ }^{3,4}$. Sin embargo, en su mayoría, son congénitas y se originan en los recesos creados durante la adherencia y la fusión de los repliegues peritoneales alrededor de la región ileocecal, y con menor frecuencia, por alteraciones de la rotación embriológica intestinal 5 .

Este tipo de hernia produce síntomas intermitentes e inespecíficos y puede simular una apendicitis aguda, por lo cual representa un reto diagnóstico prequirúrgico ${ }^{6}$. Su tratamiento es quirúrgico para reducir el contenido y cerrar el defecto herniario ${ }^{7}$.

Se reporta un caso de hernia paracecal, el cual fue un hallazgo quirúrgico incidental en el Servicio de Cirugía del Hospital de San José de Bogotá.

\section{Caso clínico}

Se trata de un hombre de 42 años de edad, sin antecedentes patológicos, quirúrgicos, ni traumáticos. Consultó por presentar un cuadro clínico de 48 horas de evolución, de dolor de tipo cólico en epigastrio y mesogastrio, irradiado a la fosa iliaca derecha; su intensidad fue progresiva hasta llegar a 7/ıo en la escala de dolor, y se asoció con cinco episodios de vómito bilioso.

En el examen físico, la frecuencia cardiaca era de 92 latidos por minuto, las mucosas estaban secas y había signos de irritación peritoneal en la fosa iliaca derecha.

En el cuadro hemático se encontró leucocitosis de I4.070 células/ $\mu \mathrm{l}$ con desviación hacia la izquierda y el resto de los parámetros estaban dentro de los límites normales. La ecografía abdominal mostró líquido libre e íleo, sin poder visualizarse el apéndice cecal.

La impresión diagnóstica inicial fue la de apendicitis aguda. Se inició reanimación hídrica con cristaloides y se administró antibioticoterapia parenteral según el protocolo de la 
institución. Posteriormente, se intervino quirúrgicamente a través de una incisión localizada de Rockey-Davis, la cual se convirtió en laparotomía ante la presencia de abundante líquido de reacción peritoneal y apéndice cecal edematoso.

Los hallazgos intraoperatorios fueron hernia paracecal y fibrosis desde el ligamento de Treitz hasta el ciego, que comprometía el mesenterio ileocecal y el apendicular; además, había múltiples adenopatías ileocecales y de yeyuno, ciego móvil y protrusión por el orificio herniario de asas yeyuno-ileales sin compromiso vascular (figuras I y 2).

Se liberó el segmento intestinal herniado, se cerró el defecto herniario y se practicó la apendicectomía. No hubo complicaciones durante el posoperatorio inmediato.

En el quinto día posoperatorio, se reintervino por una obstrucción intestinal que no mejoró con el tratamiento médico. Durante esta segunda intervención, se evidenció escaso líquido de reacción peritoneal y adherencias laxas, sin ningún otro hallazgo anormal. Se dio el egreso hospitalario cuatro días después de este último procedimiento quirúrgico $\mathrm{y}$, hasta el momento, tras 12 meses de seguimiento, ha permanecido asintomático.

En el estudio de histopatología se encontró infiltrado inflamatorio polimorfonuclear asociado con congestión vascular y hemorrágica del apéndice cecal; además, ganglios inflamatorios mesentéricos y fibrosis periapendicular y del tejido mesotelial, sin presencia de atipia.

A continuación, se analiza el tratamiento quirúrgico de un hallazgo intraoperatorio inesperado, en un paciente que -tras 12 meses de seguimiento- ha permanecido asintomático. Se hace especial énfasis en la importancia de conocer la patogénesis y el manejo de la hernia paracecal, con la finalidad de evitar complicaciones debido a sus manifestaciones clínicas poco específicas. Esta es una enfermedad en la que los síntomas no son patognomónicos.

Siempre se debe tratar de liberar y reducir el segmento herniado, corrigiendo las malformaciones asociadas, como se hizo en este paciente.

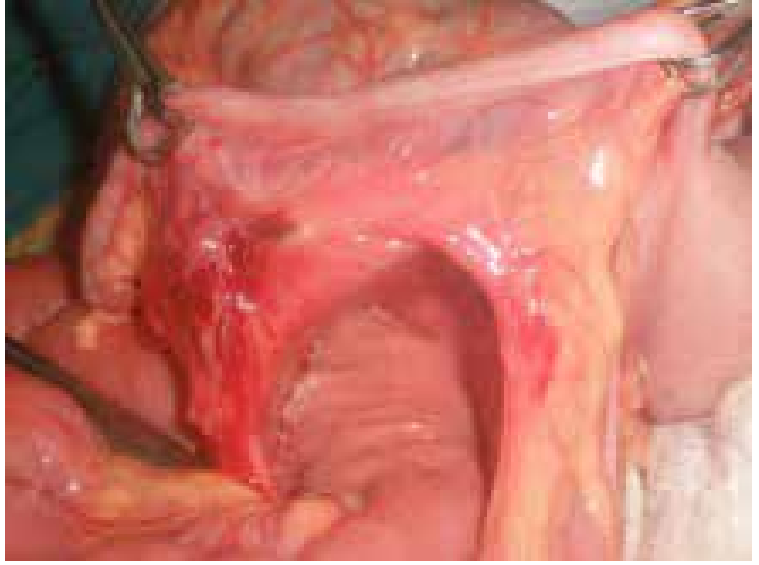

Figura 1. Orificio a través del cual protruía la hernia paracecal.

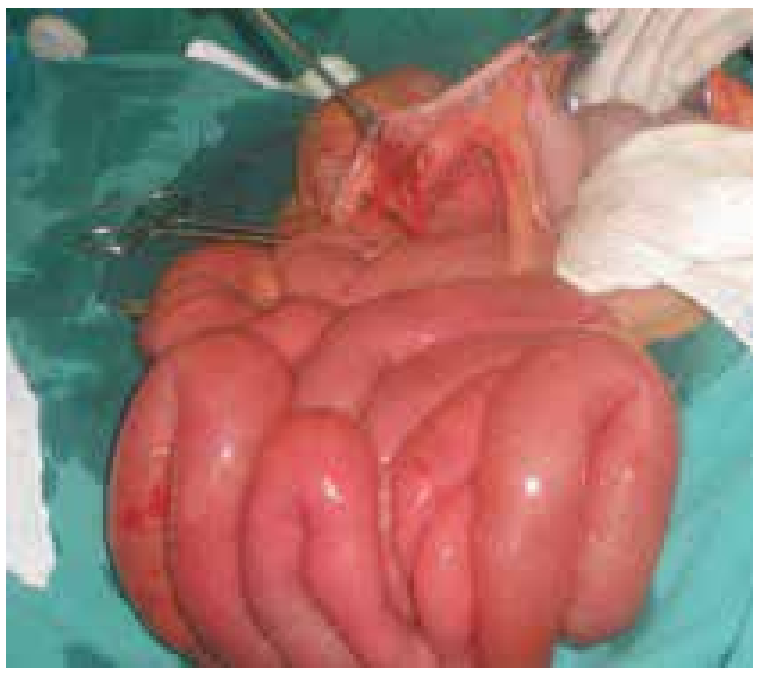

Figura 2. Hernia paracecal: dilatación proximal de asas intestinales

Se da a conocer este caso por considerarse una enfermedad poco común y un reto diagnóstico en cirugía general.

\section{Discusión}

Las hernias internas se definen como la protrusión de una víscera hueca por una apertura mesentérica o peritoneal ${ }^{8}$. Aunque las de origen congénito son las más frecuentes, se han 
descrito casos de hernias internas por formaciones peritoneales adquiridas por procesos inflamatorios, como el divertículo de Meckel complicado 9 .

Tradicionalmente, las hernias internas se clasifican como adquiridas o congénitas, y las segundas se dividen, según su localización, en retroperitoneales o intraperitoneales ${ }^{7, \mathrm{IO}}$. Según publicaciones más recientes, el tipo más común de hernia interna es la paraduodenal ( $55 \%)$, y le siguen en frecuencia las paracecales (I3\%), las del hiato de Winslow (6 a Io \%), las transmesentéricas o retroanastomóticas (6 a io \%) y las paravesicales (menos de $4 \%)^{\mathrm{II}-13}$.

Más concretamente, la hernia paracecal se clasifica en cuatro tipos, según la localización del orificio peritoneal: receso ileocecal superior, receso ileocecal inferior, receso retrocecal, y surco paracólico o fosa apendicular ${ }^{14}$; esta clasificación es utilizada con frecuencia en Japón y en los Estados Unidos. Otra de las clasificaciones que se encuentran publicadas es la propuesta por Meyer, que la divide en cuatro tipos: interna, lateral, del receso retrocecal o no clasificable ${ }^{3}$.

Teniendo en cuenta la clasificación de Endo, el sitio más común de presentación de las hernias paracecales es la fosa retrocecal, con una incidencia reportada de $74 \%{ }^{15}$. En el presente caso, se trataba de una hernia paracecal de tipo retrocecal, según la clasificación de Endo, o del receso retrocecal, según la de Meyer.

En general, las hernias internas pueden tener un saco herniario, como las transmesentéricas y las paraduodenales ${ }^{16}$, o no tenerlo, como las epiploicas o las del hiato de Winslow ${ }^{2}$.

La frecuencia de las hernias paracecales varía entre 0,2 y $0,9 \%{ }^{2,17}$ y en otras series de $\mathrm{I}-2 \%{ }^{16}$. La incidencia es mayor en hombres que en mujeres, con una relación de 3:I ${ }^{17}$; sin embargo, en algunas publicaciones recientes se informa que las tasas de incidencia no parecen variar entre hombres y mujeres ${ }^{18}$. La edad de presentación más frecuente es alrededor de los 38 años. Estas hernias pueden manifestarse como un cuadro clínico de obstrucción intestinal en o,6 a 5,4 \% de los casos $\mathrm{y}$, cuando existe estrangulación, se han reportado tasas de mortalidad hasta del $50 \%^{2,4,19,20}$.
En relación con la patogénesis, se cree que las hernias internas se originan por alteraciones en el desarrollo embriológico intestinal y la formación y ubicación de los repliegues en las capas del peritoneo dentro de la cavidad abdominal. En algunas oportunidades, pueden estar relacionadas con antecedentes de peritonitis, trauma abdominal, iatrogenia o reconstrucciones en Y de Roux. En el presente caso, podría ser por los recesos creados durante la adherencia y la fusión de los repliegues peritoneales alrededor de la región ileocecal.

Su presentación clínica es variada y puede ir desde la ausencia de síntomas o un dolor abdominal localizado, hasta un cuadro clínico de obstrucción intestinal ${ }^{21}$. Cuando se produce encarcelación con la consiguiente estrangulación del contenido herniario, la instauración de los síntomas suele ser rápida ${ }^{21,22}$. En la mayoría de los casos, su diagnóstico es difícil y su hallazgo suele ser incidental, tanto en imágenes radiológicas como en cirugía ${ }^{20-22}$.

En casos excepcionales y para hacer el diagnóstico diferencial, pueden practicarse estudios como la radiografía simple de abdomen, la tomografía abdominal con contraste, la arteriografía de vasos mesentéricos y el tránsito intestinal ${ }^{16,21}$.

La tomografía se considera el método diagnóstico de referencia en el estudio de las hernias internas ${ }^{\mathrm{II}, 23,24}$, pues permite evidenciar un conglomerado ovoide de asas yeyunales que desplazan el estómago y el colon según su localización, alteración del curso de los vasos del yeyuno o mesentéricos, presencia de un saco herniario o de puntos de transición en las asas intestinales, e íleo inespecífico ${ }^{22}$.

Por lo tanto, es de vital importancia conocer la anatomía de la cavidad peritoneal, incluyendo la localización de cada hernia, al momento de interpretar un estudio imagenológico, para garantizar un diagnóstico preoperatorio oportuno en la mayoría de los pacientes, como se ha demostrado en estudios recientes ${ }^{25,26}$.

Específicamente, los hallazgos tomográficos de una hernia paracecal pueden corresponder a la identificación de asas de intestino delgado 
localizadas en la parte posterior del ciego y del colon ascendente; además, en algunos casos, el pedículo vascular que irriga el segmento intestinal encarcelado en la hernia se ve elongado y disminuido de calibre, y las asas intestinales proximales se ven distendidas $8,16,21,27$.

El enema de bario es otro estudio imagenológico que podría considerarse en pacientes con manifestaciones clínicas leves e inespecíficas, sin signos de irritación peritoneal. Aunque no es de uso frecuente en nuestro medio, puede mostrar un grupo de asas intestinales hacia la parte posterior y lateral del ciego y del colon ascendente ${ }^{\mathrm{II}, 28}$.

En el presente caso, el paciente se sometió a cirugía con un diagnóstico de apendicitis aguda y el hallazgo de la hernia fue intraoperatorio; el único estudio previo de imágenes fue una ultrasonografía, la cual no evidenció alteraciones relacionadas con la hernia.

En contraste con las hernias internas pericecales, intersigmoideas o transmesocólicas, las paraduodenales producen isquemia o perforación con menor frecuencia ${ }^{29}$.

El pilar del tratamiento de esta enfermedad se basa en la reducción del contenido herniario y la corrección del defecto herniario ${ }^{30}$. Desde 1986 , cuando Rivkind, et al., describieron el pilar del tratamiento quirúrgico de las hernias paracecales. Este no ha cambiado, ya que comprende la liberación y reducción del contenido intestinal, la evaluación del segmento intestinal herniado, la identificación de signos de isquemia irreversible para determinar la indicación de resección intestinal con restitución del tubo digestivo, la resección del saco herniario cuando se identifica claramente, la valoración de los vasos mesentéricos y el cierre del defecto herniario ${ }^{7,14,20,31}$.

Recientemente, se ha informado sobre pacientes intervenidos mediante abordajes laparoscópicos ${ }^{15}$, que hacen posible la apertura de los orificios herniarios sin necesidad de resecar segmentos intestinales, ante la ausencia de signos de isquemia irreversible ${ }^{32-34}$. En el presente caso, el abordaje correspondió al de una apendicectomía localizada con conversión al de una celiotomía, con los hallazgos ya mencionados anteriormente.

Se presenta un caso clínico de una condición que continúa siendo un reto diagnóstico para todos los cirujanos y que se debe tener en cuenta como uno de los diagnósticos diferenciales en aquellos pacientes con manifestaciones clínicas de obstrucción intestinal de asas delgadas de etiología no clara, o de otras condiciones que puedan simular una apendicitis aguda.

\section{Conclusiones}

La presencia de una hernia interna puede llevar a diagnósticos errados o abordajes quirúrgicos inadecuados. Su diagnóstico es un reto debido al espectro de síntomas inespecíficos y a que, en la mayoría de los casos, corresponde a un hallazgo incidental durante una laparotomía o una laparoscopia.

Se debe reconocer la importancia de conocer la patogénesis de esta anormalidad y su manejo, con el fin de evitar errores técnicos y posibles complicaciones futuras, por recidiva o por compromiso vascular. A pesar de su difícil diagnóstico y baja tasa de incidencia, debe considerarse como un diagnóstico diferencial en aquellos pacientes con sintomatología de obstrucción del intestino delgado o de apendicitis aguda, y con hallazgos intraoperatorios de apéndice cecal normal o edematoso y líquido de reacción peritoneal en la región ileocecal.

Los estudios imagenológicos preoperatorios podrían ser fundamentales para su diagnóstico, teniendo en cuenta los hallazgos descritos.

El pilar del tratamiento de esta enfermedad se basa en la reducción del contenido herniario y la corrección del defecto herniario. Hoy en día, el abordaje laparoscópico es una opción adecuada que se debe considerar para el tratamiento quirúrgico de las hernias internas.

\section{Cumplimiento de normas éticas}

Consentimiento informado: los autores confirman tener el consentimiento informado del paciente para la publicación del caso.

Conflictos de interés: ninguno declarado por los autores.

Financiación: recursos propios de los investigadores. 


\section{Referencias}

I. Ghahremani GG. Abdominal and pelvic hernias. In: Gore RM, Levine MS, editors. Textbook of Gastrointestinal Radiology. Second edition. Philadelphia: Saunders; 2000. p. 1993-2009.

2. Ghahremani GG. Internal abdominal hernias. Surg Clin N Am. 1984;64:393-406. https://doi.org/IO.IOI6/s0039-6I09(I6)43293-7

3. Meyer A, Nowotny K, Poeschl M. Internal hernias of the ileo-cecal region. Erqeb Chir Orthop. 1963;44:I76-204.

4. Subasinghe D, Keppetiyagama CT, Samarasekera DN. Jejunal obstruction due to a variant of transmesocolic hernia: A rare presentation of acute abdomen. BMC Surg. 20I5;I5:57. https://doi.org/IO.II86/sI2893-0I5-005I-Z

5. Bass J Jr, Longley BJ. Paracecal hernia: Case report and review of the literature. Am Surg. 1976;42:285-8.

6. Birchley D. Ileal entrapment within a paracaecal hernia mimicking acute apendicitis. Ann R Coll Surg Engl. 2009;9:WI-3. https://doi.org/I0.I308/I47870809X400903

7. Rivkind AI, Shiloni E, Muggia-Sullam M, Weiss Y, Lax E, Freund HR. Paracecal hernia: A cause of intestinal obstruction. Dis Colon Rectum. 1986;29:752-4. https://doi.org/IO.IOO7/bfo2555327

8. Otani H, Makihara S. Laparoscopic surgery for small bowel obstruction due to paracecal hernia. Acta Med Okayama. 20I8;72:8I-4.

9. Imigo GF, Castillo FE, Rosa CE. Hernia interna secundaria a divertículo de Meckel complicado. Rev Chil Cir. 2015;67:478-9.

https://doi.org/I0.4067/So718-40262015000500003

Io. Kar S, Mohapatra V, Rath PK. A rare type of primary internal hernia causing small intestinal obstruction. Case Rep Surg. 2016;2016: 3540794.

https://doi.org/IO.II55/2016/3540794

II. Martin LC, Merkle EM, Thompson WM. Review of internal hernias: Radiographic and clinical findings. Am J Roentgenol. 2006;186:703-7. https://doi.org/I0.22I4/AJR.05.0644

I2. Selçuk D, Kantarci F, Oğüt G, Korman U. Radiological evaluation of internal abdominal hernias. Turk J Gastroenterol. 2005;16:57-64.

I3. Ghiassi S, Nguyen SQ, Divino CM, Byrn JC, Schlager A. Internal hernias: Clinical findings, management, and outcomes in 49 nonbariatric cases. J Gastrointest Surg. 2007;II:29I-5. https://doi.org/I0.I007/sII605-007-0086-2

I4. Hirokawa T, Hayakawa T, Tanaka M, Okada Y, Sawai M, Takeyama M, et al. Laparoscopic surgery for diagnosis and treatment of bowel obstruction: Case report of paracecal hernia. Med Sci Monit. 2007;13:79-82.

I5. Nishi T, Tanaka Y, Kure T. A case of pericecal hernia with a hernial orifice located on the lateral side of the cecum. Tokai J Exp Clin Med. 20II;36:7I-4.
I6. Aparício DJ, Leichsenring C, Pignatelli N, Germano A, Ferreira S, Nunes V. Transmesocolic hernia with sigmoid colon strangulation without surgical history: A series of two case reports. J Surg Case Rep. 2019;3:I-4. https://doi.org/I0.I093/jscr/rjzo73

17. Blachar A, Federle MP, Dodson SF. Internal hernia: Clinical and imaging findings in 17 patients with emphasis on CT criteria. Radiology. 200I;218:68-74. https://doi.org/IO.II48/radiology.2I8.I.roIja5368

I8. Inukai K, Tsuji E, Uehara S. Paracecal hernia with intestinal ischemia treated with laparoscopic assisted surgery. Int J Surg Case Rep. 2018;44:20-23. https://doi.org/IO.IoI6/j.ijscr.2018.02.016

19. Blachar A, Federle MP. Internal hernia: An increasingly common cause of small bowel obstruction. Semin Ultrasound CT MR. 2002;23:174-83. https://doi.org/IO.IOI6/s0887-2I7I(02)90003-x

20. Ogami T, Honjo H, Kusanagi H. Pericecal hernia manifesting as a small bowel obstruction successfully treated with laparoscopic surgery. J Surg Case Rep. 20I6;20I6:rjw020. https://doi.org/IO.IO93/jscr/rjw020

2I. Choh NA, Rasheed M, Jehangir M. The computed tomography diagnosis of paracecal hernia. Hernia. 20IO;I4:527-9. https://doi.org/I0.I007/sI0029-009-0572-4

22. McVay MR, Kokoska ER, Jackson RJ, Smith SD. The changing spectrum of intestinal malrotation: Diagnosis and management. The Am J Surg. 2007;194:712-9. https://doi.org/Io.IoI6/j.amjsurg.2007.08.035

23. Kishiki T, Mori T, Hashimoto Y, Matsuoka H, Abe N, Masaki T, et al. Laparoscopic repair of internal transmesocolic hernia of transverse colon. Case Rep Surg. 20I5;20I5:853297. https://doi.org/IO.II55/20I5/853297

24. Al-Omari MA, Al-doud MA. Simultaneous small and large bowel obstruction as a consequence of internal hernia: A case report. Int J Surg Case Rep. 2019;57:28-32. https://doi.org/IO.IOI6/j.ijscr.20I9.02.023

25. Hong SS, Kim AY, Kim PN, Lee MG, Ha HK. Current diagnostic role of CT in evaluating internal hernia. J Comput Assist Tomogr. 2005;29:604-9. https://doi.org/IO.I097/oI.rct.00ooI68326.I7363.b2

26. Zissin R, Hertz M, Gayer G, Paran H, Osadchy A. Congenital internal hernia as a cause of small bowel obstruction: CT findings in II adult patients. Br J Radiol. 2005;78:796-802. https://doi.org/IO.I259/bjr/87050272

27. Lu HC, Wang J, Tsang YM, Tseng HS, Li YW. Pericecal hernia: A report of two cases and survey of the literature. Clin Radiol. 2002;57:855-8.

28. Mathieu D, Luciani A, GERMAD Group. Internal abdominal herniations. Am J Roentgenol. 2004;183:397404 .

29. Moran JM, Salas J, Sanjuán S, Amaya JL, Rincón P, Serrano A, et al. Paramesocolic hernias: Consequences 
of delayed diagnosis. Report of three new cases. J Ped Surg. 2004;39:II2-6.

30. Tepeš M, Kirac I, Glavan E, Doko M. Internal hernias in acute abdomen: Review of literature and report of four cases. Coll Antropol. 2015;39:478-9.

3I. Kleyman S, Ashraf S, Daniel S, Ananthan D, Sanni A, Khan F. Pericecal hernia: A rare form of internal hernias. J Surg Case Rep. 2013;2013:rjso2I. https://doi.org/IO.IO93/jscr/rjso2I .

32. Omori H, Asahi H, Inoue Y, Irinoda T, Saito K. Laparoscopic paracecal hernia repair. J Laparoendosc Adv Surg Tech A. 2003;13:55-7.
33. Khalaileh A, Adileh M, Schlager A, Abu-Gazala S, Mintz Y, Rivkind AI, et al. Image of the month. Incarcerated paracecal hernia. Arch Surg. 2009;I44:975-6. https://doi.org/IO.IOoI/archsurg.2009.I7I-a

34. Kabashima A, Ueda N, Yonemura Y, Mashino K, Fujii $\mathrm{K}$, Ikeda $\mathrm{T}$, et al. Laparoscopic surgery for the diagnosis and treatment of paracecal hernia repair: Report of a case. Surg Today. 2010;40:373-5.

https://doi.org/IO.I007/s00595-009-4039-o 